PREHOSPITAL CARE

\title{
Developing alternative ambulance response schemes: analysis of attitudes, barriers, and change
}

\author{
J P Squires, S Mason
}

Emerg Med J 2004;21:724-727. doi: 10.1136/emj.2004.016923

See end of article for authors' affiliations

Correspondence to: Mr J Squires, PO Box 6490, Baslow, Bakewell DE45 1XR, UK; justin.squires@ derwentassociates.com

Accepted for publication 26 August 2004 Objectives: Alternative response schemes for emergency calls are being set up by many UK ambulance
services. The barriers to such developments from the perspective of ambulance service staff have not been formally investigated. The aims of this study are to identify attitudes and barriers to the development of alternative response schemes and ways of easing the transition as they are implemented.

Methods: South Yorkshire Ambulance Service crews and control room staff were interviewed, using South Yorkshire's paramedic practitioner (PP) scheme as a model of an alternative response scheme.

Results: 55 ambulance crew and 17 control staff were interviewed. Most $(97 \%, n=70)$ thought that the PP scheme was a good way of dealing with patients who call 999 but may not need urgent transport and hospital treatment. The perceived effect of the PP scheme on traditional ambulance service duties was equally divided between a third who thought there had been no effect, a third who perceived an improvement, and a third a deterioration. Recurrent difficulties with the scheme were found relating to the AMPDS methodology of ambulance dispatch, and ambulance service performance targets.

Conclusions: Flexibility of AMPDS and dispatch targets will need to be reviewed to permit the successful implementation of alternative responses to 999 calls. Careful consideration needs to be given to communicating the aims and value of such schemes to all staff and ensuring a common understanding of, and commitment to, a shared vision. The effect of implementation on the remaining service function must be well planned.
$\mathrm{T}$ raditionally UK ambulance services have transported patients requesting emergency assistance to hospital for assessment and treatment. The development of extended training in the mid-1980s led to the role of the paramedic and brought some treatment out of the hospital to the patient. Driven by the increasing number of emergency medical admissions, demands on the ambulance service and documents such as Reforming Emergency Care by the Department of Health $^{1}$ and $A$ Life in the Fast Lane by the Audit Commission, ${ }^{2}$ some ambulance services have started developing alternative response schemes whereby certain groups of patients with a defined set of conditions are assessed, and potentially definitively treated at home, by ambulance service personnel. ${ }^{3}$ Such developments have been supported by the Joint Royal Colleges Ambulance Liaison Committee (JRCALC). ${ }^{4}$ Internationally, similar concepts are being considered and developed and issues relating to them described..$^{5-9}$

One such alternative response scheme is the Sheffield paramedic practitioner (PP) scheme set up through the collaboration of the South Yorkshire Ambulance Service (SYAS), the Emergency and Care of the Elderly Departments of Sheffield's Northern General Hospital (NGH), and the Sheffield Community Social Services Team. The scope of the scheme has already been defined, ${ }^{10}$ but in summary it is aimed at patients over 60 years of age, in the Sheffield area, who call 999 with minor acute conditions such as falls, limb or head injury.

The PPs are activated either by the control room if the call is identified as being appropriate through the advanced medical priority dispatch system (AMPDS) or on the attendance of a traditional emergency ambulance if the crew find that the incident fits the PP scheme criteria. A PP response is one PP in a paramedic car, in place of the traditional emergency ambulance response. Patients are assessed at home by PPs with additional training to emergency nurse practitioner level and where possible they are also treated at home. If further investigations such as radiography are required then transfer to the emergency department (ED) is arranged. Normally a further vehicle (urgent crew $^{*}$ or emergency ambulance) is arranged to transfer the patient unless they are sufficiently mobile and able to be carried in the PP car, in which case this is also possible. This scheme has been operational daily between 0800 and 2000 since October 2002, and is currently part of a randomised controlled trial (RCT) to evaluate its effect. This trial has randomised the service delivery into operational "on" weeks and non-operational "off" weeks. The results of this RCT will be important as there has not yet been a significant body of research evaluating the clinical and cost effectiveness of such schemes. ${ }^{11}$

As ambulance services are setting up such schemes including the development of the emergency care practitioner role $^{4}$ the importance of evaluating attitudes, barriers, and change management issues is vital to ensuring their success. This means careful consideration must be given to identify the key stakeholders, gain their agreement, and anticipate barriers they may either experience or perceive. ${ }^{12}$

This study aims to assess the attitudes of SYAS crew members and control room staff to the PP scheme, with the following objectives:

- to identify barriers to the development and real time activation of alternative response schemes, using the

Abbreviations: PP, paramedic practitioner; SYAS, South Yorkshire Ambulance Service; AMPDS, advanced medical priority dispatch system; RCT, randomised controlled trial; EMT, emergency medical technician; EMD, emergency medical dispatcher; NGH, Northern General Hospital

*Urgent crews primary role is to transport patients with non-life threatening conditions to hospital, usually having first been seen by their general practitioner. 
knowledge and understanding of the Sheffield PP scheme as an example;

- to highlight key change management areas that should be considered by other services setting up such schemes.

\section{METHODS}

During January and February 2004, the views of ambulance crews (urgent crew, emergency medical technician (EMT) or paramedic) and control staff (emergency medical dispatchers (EMD) and dispatch managers) were sought using semistructured interviews. All interviews were administered by the same investigator (JS) who had no involvement in SYAS or the PP scheme. The format of the interviews had some common parts between the groups and some areas that were group specific. It was known that the call identification rate for eligible calls by EMDs alone was low. Previous work has shown that control room staff identified about $37 \%$ of eligible calls. This should be compared with an identification rate of $>90 \%$ when a PP is in the control room scanning and picking up all calls. ${ }^{13}$ Given this low rate of identification the control staff group were provided with a test of patient scenarios for them to decide which would be suitable for referral to the scheme.

The crews serving Sheffield were approached and invited to participate in the study after patient handover at the NGH $\mathrm{ED}$, at the completion of one interview the next available crew were selected as the next to be interviewed. The interviews were mainly carried out during normal working hours, as they were conducted over a four week period the rotation of shift patterns ensured that no specific group of staff were excluded. Although all ambulances were double crewed the interviews were undertaken with staff individually. The control staff were approached and interviewed while on duty at the SYAS control centre. Verbal consent to take part was gained at the initial approach to each participant.

Demographic details recorded included occupation and total time with the ambulance service. A combination of closed questions, free text comments and visual analogue scales were used to capture data on the following areas:

- level of awareness of the PP scheme;

- thoughts on the PP scheme;

- knowledge of the PP research project;

- general views on SYAS.

Copies of the forms completed in the interviews are shown in appendices 1 and 2 (available to view on the journal web site http://www.emjonline.com/supplemental).

Data were entered onto a Microsoft Access database, frequencies and means were analysed using SPSS for Windows (version 12.0.0).

The study was approved by the North Sheffield Research Ethics Committee.

\section{RESULTS}

\section{Ambulance crew}

There are 120 ambulance crew covering Sheffield, 55 (46\%) were interviewed, comprising six urgent crew, 17 EMTs, and 32 paramedics. No one declined to be interviewed. The mean number of years service of those interviewed was 14 , ( $\mathrm{SD}=8.6$, range 1 to 39 years.)

All those interviewed were aware of the PP scheme. Table 1 shows the proportion of staff describing specific key areas of the scheme.

Most $(96 \%, \mathrm{n}=53)$ thought that the PP scheme was a good way of dealing with patients who call 999 but may not need urgent transport and hospital treatment. This reply was often
Table 1 Grouped responses to "Tell me what you know about the scheme"

\begin{tabular}{lllr}
\hline Area & Scheme descriptor & $\begin{array}{l}\text { Number of } \\
\text { staff } \\
\text { describing }\end{array}$ & $\begin{array}{l}\% \text { Of staff } \\
\text { describing }\end{array}$ \\
\hline 1 & Over 60s & 54 & 98 \\
2 & 999 calls (+urgent not seen) & 1 & 2 \\
3 & Minor injuries/conditions & 25 & 45 \\
4 & Home treatment & 41 & 75 \\
5 & ED fast tracking, for example, to & 11 & 20 \\
6 & radiography & 10 & 18 \\
7 & Involves paramedics with & additional training and skills & 44 \\
8 & Can include social aspects of care & 24 & 4 \\
& Calls referred to the scheme by & 2 & \\
\hline & other ambulance crews & & \\
\hline
\end{tabular}

given with the caveat that full cover must be maintained to deal with life threatening emergencies.

Eighty nine per cent $(n=49)$ thought there were other groups of patients who should receive this type of service. Most of those thought the range should extend to cover all age groups of patients presenting with minor injury or illness. It was also commented that the scheme could be extended to patients who find it difficult to leave their homes for other reasons such as mental health problems or mobility difficulties.

While two thirds $(67 \%, \mathrm{n}=37)$ of respondents had made referrals to the scheme more than five times, $13 \%(n=7)$ had never made a referral.

Considering problems making referrals to the scheme, 29\% $(\mathrm{n}=16)$ said they had experienced no problems. Most problems were with availability of the scheme:

- $47 \%(n=26)$ had experienced problems with referral when the PPs were not operational because of the RCT "off" weeks;

- $11 \%(n=6)$ had highlighted problems attempting to make referrals outside the current operational hours of the scheme;

- $5 \%(n=3)$ had encountered a problem with the exact referral criteria for the scheme;

- $5 \%(n=3)$ had experienced internal difficulties within the organisation of the scheme.

The crews were asked if they had received adequate information about the scheme, $82 \%(n=45)$ thought they had. Comments from those who felt they had not had enough related to general information overload within the service.

The perceived effect of the PP scheme on traditional ambulance service duties was equally divided between a third who thought there had been no effect, a third who perceived an improvement and a third a deterioration. Nearly all those who thought that the scheme had a detrimental effect on normal ambulance duties stated that this was because the PPs were still on the normal paramedic rota at their respective ambulance stations but were unavailable to undertake normal duties. Other reasons given for this perceived deterioration were:

- the potential for the PPs to become de-skilled in the management of major trauma and cardiac arrest situations as they are likely to see these cases less frequently;

- the perception that training of those outside the scheme was being reduced. 
Most of those who thought that the PP scheme had a positive effect on traditional ambulance duties said this was because it enabled emergency crews to be left to concentrate on dealing with more serious emergencies as they could leave the PPs to deal with the minor cases.

Eighty per cent $(n=44)$ thought that all paramedics should be trained to the same level as the PPs. However this was usually qualified by concern about the delivery of frontline ambulance services should such training be put in place.

\section{Control staff}

Of the 39 control room staff, 17 (44\%) were interviewed, comprising 11 EMDs and six dispatch managers. No one declined to be interviewed. The mean number of years service of those interviewed was 2.9 , ( $S D=2.6$ years, range 3 months to 8 years.) All thought that the PP scheme was a good way of dealing with patients who call 999 but may not need urgent transport and hospital treatment.

A recurring theme throughout the interviews with the EMDs was that of the standard AMPDS. This is the system used to prioritise and manage calls within the ambulance service. It was found that currently it has not been modified in any significant way to accept the PP initiative and that the present method of identifying calls as appropriate for a PP response needs to be done early in the process of taking the call. This was reported to be awkward for the EMDs, in that if it is not initially apparent that the call is eligible and this is only found out during the call, an amount of back tracking is then needed to enter this at the beginning of the record. As this is a potentially time consuming process that the EMDs are currently not given any acknowledgement of in the monitoring of their performance, it became clear that they default to not identifying calls for the PP scheme if under pressure.

Both control room staff groups scored highly on their identification rate of patient scenarios that should be considered for referral to the scheme. The mean score of the EMDs was $83 \%$ and $90 \%$ for the despatch managers. These scenarios are shown in appendix 2 .

None said they had experienced difficulties with the possible additional work or responsibility of referring to the PP scheme.

The despatch managers felt a specific valuable outcome from the scheme was a reduction in the pressure to dispatch ambulances immediately when the calls were flagged by the EMDs as appropriate for PP, easing pressure on them and the rest of the service.

\section{DISCUSSION}

Overall most respondents felt that they had enough information about the scheme and that it was good for patients. There were however a number of areas identified that require consideration in the development of such alternative responses. These provide an insight into how the next generation of alternative responses may be received.

\section{Barriers to development of alternative responses Identification of eligible calls and referral}

Referral to the scheme was a problem for both crews and control room staff, however this was mainly because of the effects of the RCT, which has made the scheme only operational on certain weeks as a method of randomisation. These concerns may partially account for the low identification of eligible patients by the control room staff in reality, compared with their knowledge level as demonstrated by their responses to the scenarios in the interviews where they scored highly. However, another key factor is that the AMPDS has not been designed or is not used with alternative response schemes in mind. Staff are monitored according to exact adherence to their protocols and the speed with which calls are completed, linking to the overall attainment of service ORCON standards. So there may be little motivation to re route calls to an alternative response, if this takes additional time to do, especially if there is a queue of 999 calls waiting to be answered.

This creates a systematic problem in the development of alternative responses. It is suggested that flexibility needs to be introduced into AMPDS to allow alternative referrals to be considered at any time during the progress of the call. If there is a possibility of the patient being appropriate for such referral, the performance monitoring systems should be changed so EMDs and ambulance services are not penalised while a specific line of questioning is pursued to confirm eligibility and appropriateness of dispatch.

\section{Ambulance dispatch targets}

Ambulances are dispatched to emergency calls virtually as soon as the location of the incident is confirmed, in accordance with one of the key steps to improving ambulance service performance as identified by the Department of Health. ${ }^{14}$ Dispatch usually takes place before details needed to identify eligibility for the scheme, particularly the age of the patient, have been entered on the system. Hence to transfer the call to an alternative response may often entail additional work standing a crew down and re routing the call. The drive to meet time response targets therefore does not currently sit happily with increasing the pathways of care available to patients, unless ways can be found to achieve both simultaneously. This situation is exacerbated by current ambulance service assessment targets ${ }^{15}$ that place no value on the use and development of alternative responses. Other developmental projects such as the West Midlands Ambulance Service emergency nurse advisors have also found their implementation limited by constraints relating to target times. ${ }^{16}$ As alternative response schemes may be perceived to remove resource from the ambulance services core functions, which are included in the monitoring for the targets, they may even be considered to be detrimental to the service. Such standards need to be reviewed to facilitate the successful development of alternative response schemes.

\section{Change management areas that should be considered by ambulance services setting up alternative response schemes}

Communication and commitment to change

Although most respondents felt that they had enough information about the scheme, the detailed knowledge of the key descriptors of the scheme was low. Important features such as fast tracking PP patients through the ED, for example direct to radiography, were only mentioned by $20 \%(n=11)$. Such a factor may have an impact on referral rates because of some crews' lack of understanding of how the scheme may benefit patients. This emphasises that good communication and training throughout the service to promote understanding and commitment are important success factors. Indeed uncertainty or lack of knowledge about change, and lack of a shared vision and common direction are well recognised barriers to successful implementation of change. Removing the uncertainty by ensuring that the objectives and the reasons for the change are understood and agreed by all concerned through communication and involving people is key to successful implementation. ${ }^{17}{ }^{18}$

\section{Effect on emergency ambulance functions}

The posts of PP have been filled by staff who would otherwise have been doing front line ambulance work. At a time when the number of emergency calls is rising, ${ }^{2}$ an effective 
reduction in the number of front line staff has caused some bad feeling among the crews, indeed almost all of those who said the scheme had a worsening effect on normal ambulance duties did so for this reason. One paramedic summed up their attitude to the scheme as "everyone gains, the patients, a\&e... apart from E [emergency] crews." Clearly careful consideration is needed to identify and recruit personnel with the right skills, but perhaps it is equally important to consider the impact on the remaining operations of moving staff to projects such as this. This is an inherent problem with such change, indeed the literature on projects and their implementation focuses almost exclusively on selecting personnel for the project, as compared with considering the impact of removing key members of staff on the rest of the organisation. ${ }^{12}$

As most respondents thought that all paramedics should be trained to this level there are no identified barriers relating to having and using the skills themselves, but resistance relating to the possible compromise of the front line ambulance function. This resistance may be based on the premise that the operational organisation of the front line service does not change. The status quo is unlikely to remain as all areas of ambulance activity are becoming subject to critical review and organisational change will be needed. ${ }^{19}$ People who ignore or do not anticipate change clearly hinder the successful implementation of change that the organisation wants to achieve. ${ }^{20}$

\section{Limitations}

This study was limited by the number of staff available for interview during the study period and the effects of the RCT on the PP scheme as described above. The scenarios to test referral criteria knowledge were only carried out on control staff, if these had been carried out with the ambulance crews in addition, a comparison could have been made between the two groups. Although this study was only carried out on one ambulance service, the authors believe the conclusions below are generic issues with wider application.

\section{CONCLUSIONS}

Flexibility of AMPDS and dispatch targets will need to be reviewed to permit the successful implementation of alternative responses to 999 calls. Careful consideration needs to be given to communicating the aims and value of such schemes to all staff and ensuring a common understanding of, and commitment to, a shared vision. The effect of implementation on the remaining service function must be well planned.

\section{CONTRIBUTORS}

The study idea was conceived by SM and developed by both authors. Data were collected by JS, both authors contributed to its interpretation. JS wrote the first draft of the paper, both authors contributed to the final draft.

\section{ACKNOWLEDGEMENTS}

The authors thank the members of South Yorkshire Ambulance Service who participated in the interviews.

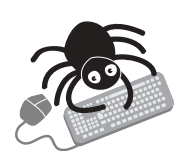

Appendices 1 and 2 are available on line (http:// www.emjonline.com/supplemental).

\section{Authors' affiliations}

J P Squires, Faculty of Medicine, University of Sheffield, Sheffield, UK S Mason, Emergency Department, Northern General Hospital, Sheffield and Medical Care Research Unit, University of Sheffield, Sheffield, UK Funding: none.

Conflicts of interest: none declared.

\section{REFERENCES}

1 Department of Health. Reforming emergency care. London: Department of Health, 2001.

2 Audit Commission. A life in the fast lane: value for money in emergency ambulance services. London: Audit Commission 1998.

3 Snooks H, Kearsley N, Dale J, et al. New models of care for 999 callers with conditions that are neither life threatening nor serious: results of a national survey. Prehospital Immediate Care 2000;4:180-2.

4 Joint Royal Collages Ambulance Liaison Committee. The future role and education of paramedic ambulance service personnel. London: JRCALC, 2000.

5 Snooks H, Dale J, Kearsley N, et al. Development and impact of emergency ambulance "Treat and Refer" protocols for non-serious 999 patients. London: London Ambulance Service, 2001.

6 Schmidt T, Atcheson R, Federiuk C, et al. Evaluation of protocols allowing emergency medical technicians to determine need for treatment and transport. Acad Emerg Med 2000; 7:663-9.

7 Hauswald M. Can paramedics safely decide which patients do not need ambulance transport or emergency department care? Prehospital Emergency Care 2002;6:383-6.

8 Silvestri S, Rothrock SG, Kennedy D, et al. Can paramedics accurately identify patients who do not require emergency department care? Prehospital Emergency Care 2002;6:387-90.

9 Kamper M, Mahoney BD, Nelson S, et al. Feasibility of paramedic treatment and referral of minor illnesses and injuries. Prehospital Emergency Care 2001; 5:371-8

10 Mason S, Wardrope J, Perrin J. Developing a community paramedic practitioner intermediate care support scheme for older people with minor conditions. Emerg Med J 2003;20:196-8.

11 Snooks HA, Dale J, Hartley-Sharpe C, et al. On-scene alternatives for emergency ambulance crews attending patients who do not need to travel to the accident and emergency department: a review of the literature. Emerg Med J 2004;21:212-15.

12 Briner W, Geddes M, Hastings C. Project leadership. Aldershot: Gower, 1992.

13 Knowles E, Mason S, Wardrope J. Challenges in triggering ambulance service alternative responses. Annual scientific meeting of the Faculty of Accident and Emergency Medicine, London, Nov 2003 (poster presentation).

14 Department of Health. Ambulance improvement checklist. http:// www.doh.gov.uk/emergencycare/ambulancechecklist.htm (accessed 12 Feb 2004).

15 Commission for Health Improvement. What $\mathrm{CHI}$ has found in: ambulance trusts, sector report. http://www.chi.gov.uk/eng/cgr/ambulance/report03/ ambulance_report.pdf (accessed 21 Feb 2004).

16 Robertson-Steel I. "Reforming emergency care": the ambulance impact. A personal view. Emerg Med J 2004;21:207-11.

17 Kanter RM, Stein BA, Jick TD. The challenge of organizational change. New York: The Free Press, 1992.

18 Carnall CA. Managing change in organizations. 3rd ed. Harlow: Prentice Hall, 1999.

19 Nicholl J, Turner J, Martin D. The future of ambulance services in the United Kingdom. Sheffield: Medical Care Research Unit, ScHARR, University of Sheffield, 2001.

20 Johnson S. Who moved my cheese? London: Vermillion, 1998. 